\title{
$(4)$
}

UNIVERSIDAD PERUANA DE CIENCIAS APLICADAS

FACULTAD DE CIENCIAS DE LA SALUD

PROGRAMA ACÁDEMICO DE MEDICINA

\section{FRECUENCIA Y FACTORES ASOCIADOS A LA INFECCIÓN POR VIH EN COMUNIDADES SHIPIBO- KONIBO DE LA REGIÓN LORETO - PERU} TESIS

Para optar el título profesional de Médico Cirujano

\section{AUTOR(ES)}

Valdez Arellano, Marycielo Alesandra (0000-0002-5854-0187)

Mendoza Hayashida, Yomaira Harumi (0000-0001-7075-9715)

\section{ASESOR (ES)}

Soto Tarazona, Alonso Ricardo (0000-0001-8648-8032)

Lucchetti Rodriguez Aldo Javier (0000-0003-1150-168X) 


\section{AGRADECIMIENTOS}

Las pruebas de tamizaje fueron financiadas por la ONG AHF Help Foundation junto a la Dirección Regional de Salud de Ucayali.

Agradecemos a la Dra. Miriam Pajuelo, médico del Instituto Nacional de Salud del Perú y a Giovanni Flores Arancibia, jefa del Centro de Salud de Contamana. Asimismo, agradecer al grupo de médicos del Centro de Salud Contamana que realizaron los viajes para la obtención de fichas de tamizaje. 


\section{RESUMEN}

Antecedentes: La frecuencia de infección por VIH en poblaciones amazónicas muestra resultados disimiles. La comunidad Shipibo-Konibo es la segunda etnia amazónica peruana en número y existen reportes previos de frecuencias de infección por VIH elevadas en las regiones colindantes. Sin embargo, no existen estudios que hayan evaluado la frecuencia de infección en esta etnia.

Objetivos: Evaluar la frecuencia y factores asociados a la presencia de infección por VIH en la comunidad Shipibo-Konibo de la provincia de Ucayali en el Departamento de Loreto.

Métodos: Estudio transversal analítico basado en el análisis de los registros obtenidos durante el proceso de búsqueda activa de VIH realizado en el Centro de Salud de Contamana en visitas de atención durante marzo 2017- marzo 2018. El diagnóstico definitivo se basó en dos pruebas rápidas positivas distintas.

Resultados: Se obtuvo información de 461 pobladores, con una prevalencia de $11.49 \%$ de infección por VIH. El 63.9\% de tamizados fueron de sexo femenino y la mediana de edad fue de 27 años. Se tamizaron 45 gestantes, de las cuales 12 (27\%) fueron VIH (+). En el análisis multivariado la edad $(\mathrm{OR}=0.93)$, baja de peso $(\mathrm{OR}=12.77)$, y diarrea $(\mathrm{OR}=36.41)$ se asociaron significativamente a la infección por VIH.

Conclusiones: La población perteneciente a la etnia Shipibo-Konibo estudiada presentó una alta prevalencia de infección por VIH, muy por encima del promedio nacional. Se deben implementar medidas urgentes tomando el contexto cultural de esta población para evitar el desarrollo de una epidemia de infección por VIH en esta comunidad.

"PALABRAS CLAVE: VIH; Indígenas; Perú; Salud pública; Factores de riesgo" 


\section{ABSTRACT}

Background: The frequency of infection by HIV in Amazonian communities shows dissimilar results in different, evaluated communities. The Shipibo-Konibo community is the second Amazonian ethnic groups in Peru and has been reported for high proportions of HIV infections in closing regions. However, there are no studies that have evaluated the frequency of this infection in this ethnic group.

Objectives: Evaluate the frequency and associated factors with the presence of HIV infection in the Shipibo-Konibo community of the province of Ucayali in the Department of Loreto.

Methods: A transversal analytical study is presented based on the screening charts obtained during the active surveillance conducted by the Contamana Health Center in care visits from March 2017 to March 2018. The final diagnosis of HIV infection was based on two positive rapid tests.

Results: Data was obtained from 461 inhabitants, with a prevalence of $11.49 \%$ of HIV infection. The $63.9 \%$ were female and the median age was 27 -year-old. Forty-five pregnant women were tested, and 12 of them $(27 \%)$ were HIV $(+)$. In the multivariate analysis, we found that age $(\mathrm{OR}=0.927)$, low weight $((\mathrm{OR}=12.771)$ and diarrhea $(\mathrm{OR}=36.409)$ were significantly associated with the presence of HIV infection.

Conclusions: The population of the Shipibo-Konibo ethnic group showed a high prevalence of HIV infection (11.49\%), above to the national average. We believe that urgent measures should be implemented based on cultural context of this population to avoid the development of an epidemic of HIV infection in this community.

“KEY WORDS: HIV; Indigenous population; Peru; Public health; Risk Factors" 
Tabla de contenido

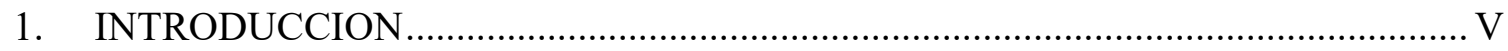

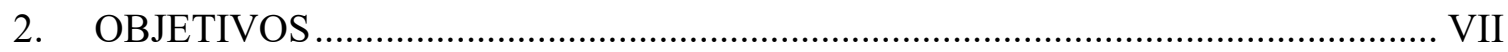

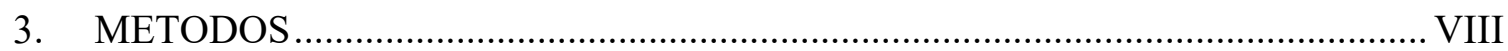

3.1 Población y Muestra ............................................................................................ VIII

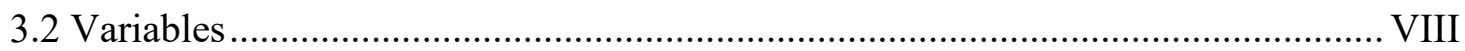

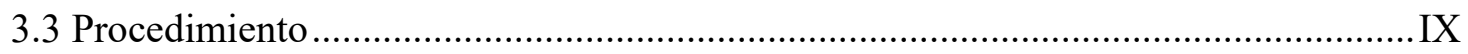

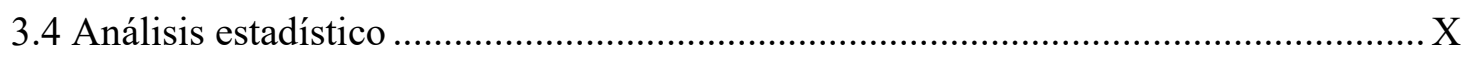

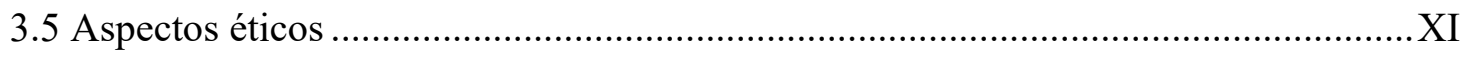

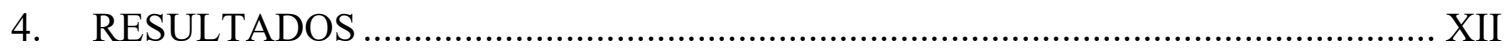

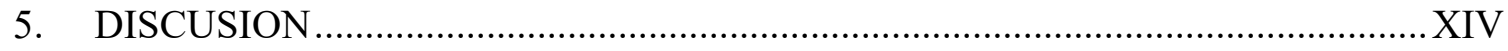

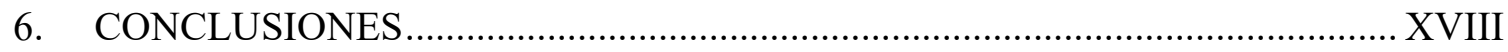

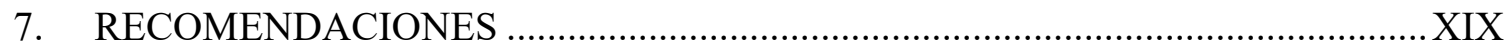

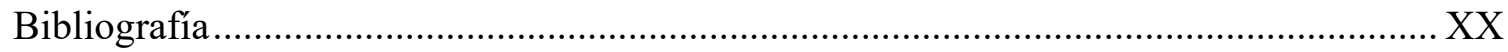

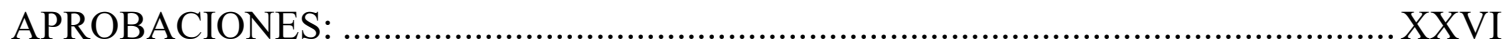

\section{Índice de Tablas}

Tabla 1: Características sociodemográficas de la población estudiada según status de seropositividad a VIH. Provincia de Ucayali, Perú. 2017 -2018............................XXII

Tabla 2 : Modelo multivariado de variables significativas, Provincia de Ucayali, Perú.

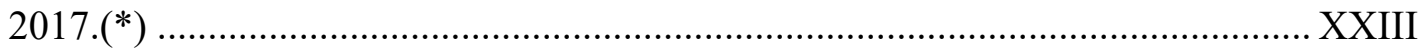

Tabla 3 : Modelo multivariado de variables significativas, Provincia de Ucayali, Perú.

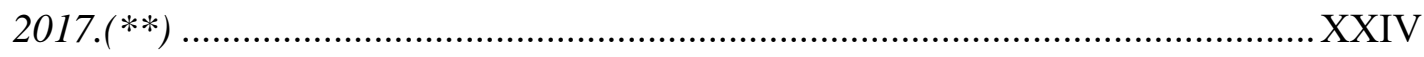

\section{Indice de Figuras}

Figura 1: Flujograma para diagnóstico de infección por VIH, Ucayali, Loreto-Perú 2017 XXV 


\section{INTRODUCCION}

Latinoamérica es un continente compuesto de diferentes grupos étnicos con diferentes costumbres y localizados en distintos lugares. En las últimas tres décadas se ha tratado de establecer las características epidemiológicas de la infección de VIH. Existen estudios donde reportan la aparición del VIH en comunidades indígenas de Latinoamérica. En Venezuela, un estudio en indígenas del Orinoco reveló que existía un $9.5 \%$ de prevalencia en las comunidades indígenas Warao (1). Lo mismo se ha observado en Brasil, donde se evaluaron las comunidades Waiamapi y Tiriyo, en las cuales presuntamente no existía esta infección, pero se encontró pacientes con VIH. Perú es un país en el cual existen diferentes etnias y grupos raciales. Gran parte de ellos son grupos indígenas que se extienden desde la sierra a la selva peruana. Un grupo importante y el segundo en cantidad de la población indígena peruana, es el Shipibo Konibo que se encuentra en la amazonia peruana (2). A pesar de esto, no se tiene información sobre el estado de salud integral de esta comunidad y el último Análisis de Situación de Salud Shipibo data del año 2002. Un aspecto importante a tener en cuenta son las enfermedades infecciosas emergentes, las cuales van en aumento por las costumbres de la población y la geografía de la comunidad. Entre estas, son relevantes las infecciones de transmisión sexual, incluyendo la infección por VIH (1). En Perú, se desarrollaron estudios en comunidades nativas desde el año 2004, cuando se reportó el primer caso de infección por VIH en nativos amazónicos. En el estudio de Ormache et al se presenta una población que abarca 6 grupos indígenas diferentes, los cuales se encuentran en distintas zonas del Perú (Loreto: Kandozi, Shapra y Shiwilo, Ucayali: Shipibo Konibo, Cusco: Matsigenka, Junin: Ashaninka y Pasco: Ashaninka), siendo la población de estudio las gestantes y sus parejas. En éste, se encontró prevalencia de VIH en gestantes de $0.16 \%$ y en sus parejas masculina de $0.29 \%$. (3)

El estudio de Zavaleta et al se centra en la población Chayahuita, la cual está ubicada en Alto Amazonas; se contó con una población de 162 habitantes, el tamizaje de VIH se dió con 2 pruebas y se apoyó en un traductor para realizar las entrevistas. En éste, la prevalencia de VIH fue $7.5 \%$ (6 de 80$)$. (4) 
Durante los años 2015 y 2016 en la Localidad de Contamana, capital de la provincia de Ucayali, departamento de Loreto, se realizaron campañas de tamizaje por el día mundial del SIDA registrándose 40 casos de VIH. Esta cifra correspondería a una prevalencia de 200 por cada 100,000 habitantes, dando una prevalencia de $2 \%$, la cual es una tasa alta en comparación a la seroprevalencia peruana (5). Esta tasa podría involucrar tanto a pobladores de la ciudad como a nativos Shipibos-Konibo de la zona (comunicación personal). Pese a ello, no se encontró información sobre el estado de la infección de VIH en la comunidad Shipiba que reside en Contamana. Al ser una población poco estudiada y vulnerable, puede presentar factores de riesgo para el contagio de VIH diferentes a los factores clásicamente reconocidos en las poblaciones de riesgo y similares a aquellos encontrados en otras poblaciones indígenas.(1) (6)

Se han realizado recientes estudios en los cuales se comienza a analizar posibles factores de riesgo que afecten esta población. Se postula que características tales como inicio sexual temprano (edad promedio 13 años) y múltiples parejas sexuales podrían aumentar el riesgo de infección de VIH. También se plantea que los roles de género son un factor importante, siendo la decisión del hombre la que prima en circunstancias como el uso de preservativo y de mantener diferentes parejas sexuales fuera del matrimonio. Otra característica que afecta a esta población es el uso del río como medio de transporte, en donde el intercambio de mercadería y también el intercambio de servicios sexuales se hacen presentes (7).

Nuestro estudio difiere a previos estudios porque es el primero en enfocarse solo en la comunidad Shipibo Konibo peruana. Al tener pocos estudios y con porcentajes de seroprevalencia que difieren, es necesario un estudio que se centre en la comunidad ShipiboKonibo, posterior al cual, se deben realizar estudios estructurales y de características específicas de la misma para realizar un plan de prevención adecuado a los factores de riesgo específicos que presenta. 


\section{OBJETIVOS}

El objetivo del estudio fue determinar la prevalencia de la infección por VIH en la población Shipibo Konibo de la provincia de Ucayali del Departamento de Loreto y explorar posibles factores asociados a la infección por VIH en esta comunidad. 


\section{METODOS}

\subsection{Población y Muestra}

Se realizó una investigación transversal analítica, en la población Shipibo-Konibo de la provincia de Ucayali, región Loreto en la Amazonia Peruana, desde marzo 2017 hasta marzo de 2018. En base a un nivel de confianza del 95\%, una frecuencia esperada de 5\% (4), una precisión de $+/-2 \%$ y basada en una población de 20,000 habitantes, el número de muestra estimado fue de 419 participantes. Se consideró un máximo de $10 \%$ de fichas no respondidas o incompletas, por lo que el total final de la muestra fue de 460 participantes. Dicha muestra fue calculada con el fin de hallar prevalencias, no se realizó un cálculo considerando pruebas de hipótesis dada la naturaleza exploratoria del componente analítico del presente estudio. Se incluyó a pobladores mayores de 18 años de la comunidad Shipibo-Konibo de la provincia de Ucayali que accedieron a responder a la encuesta de tamizaje y contaron con fichas de evaluación correctamente llenadas. Asimismo, se excluyeron a pacientes con diagnóstico previo de VIH.

\subsection{Variables}

La variable principal de este estudio es la positividad para el Virus de inmunodeficiencia Humana (VIH), esta variable fue medida con las pruebas rápidas Rapid HIV I\&II Test (Lumiquick ${ }^{\circledR}$ ) y HIV $1 / 2$ Ab Plus Combo Rapid Test (ARIA®). $\left.{ }^{\circledR}\right)$. En el caso de Lumiquick la prueba presenta un 99.6\% de especificidad y $99.7 \%$ de sensibilidad. La prueba se realiza con sangre completa, se apertura la prueba, se coloca en una superficie plana y horizontal, se coloca dos gotas de sangre total con el dispensador que incluye el kit y se espera 20 minutos después de colocarla hasta la lectura, lecturas luego de los 30 minutos pueden generar falsos positivos. Las pruebas se deben mantener en temperaturas de $4-30^{\circ} \mathrm{C}$ (8). Asimismo, en el caso de HIV 1/2 Ab Plus Combo Rapid Test, dicha prueba presenta una sensibilidad relativa del 100\% y especificidad relativa de $100 \%$; esta se puede realizar con plasma, suero o sangre completa, en el caso del presente estudio se realizó con sangre completa, se dispone de una gota de 
sangre completa en el circulo indicativo de muestra y dispensar una gota de diluente de muestra inmediatamente, los reactivos pueden ser leídos en 15 minutos, asimismo, resultados positivos pueden ser visibles desde el primer minuto, todos los reactivos deben estar a temperatura ambiente $\left(15-30{ }^{\circ} \mathrm{C}\right)$ antes de usarlos. (9)

El resultado fue VIH confirmado si ambas pruebas fueron positivas. Si una prueba fue positiva y la otra negativa, se repitió la misma. $\mathrm{Si}$, ambas pruebas vuelven a ser discordantes se procede a realizar carga viral. (Figura 1). El uso de este algoritmo ha sido certificado por CDC, aumenta el valor predictivo positivo de la prueba y aumenta el porcentaje de personas que luego de haber resultado positivos en la prueba es derivado a un centro de salud para su tratamiento. También se observa que al usar el algoritmo solo se espera un falso positivo por cada 500,000 sujetos testeados $(0.0002 \%)(10)$

Asimismo, se registraron como variables independientes: sexo, edad, gestación, ocupación, estado civil, edad de inicio sexual, consumo de sustancias toxicas (alcohol, tabaco, drogas), conductas de riesgo, tipo de relaciones sexuales, número de parejas estables, presencia de ITS y signos sugerentes de infección por VIH.

\subsection{Procedimiento}

El tamizaje se desarrolló en la provincia de Ucayali, el cual comprende 6 distritos, de los cuales no se estudió el distrito donde se ubica la capital de la provincia (Contamana), en los cinco distritos restantes se incluyeron 6 comunidades: Pampa Hermosa (Distrito de Pampa Hermosa), Inahuaya (Distrito de Inahuaya), Juancito y Tierra Blanca (Distrito de Sarayacu), Tiruntan (Distrito Padre Márquez), y Orellana (Distrito Alfredo Vargas Guerra). Estos distritos fueron elegidos por la estrategia de control de VIH del puesto de Salud de Contamana, debido a que, comparando todas las comunidades indígenas, estas tenían mayor cercanía al rio Ucayali, cada comunidad fue visitada aproximadamente 2 veces a lo largo del estudio y fue por 5 miembros de personal de salud (médico, obstetra, dos técnicos de laboratorio y una enfermera), las visitas fueron mensuales debido a los largos recorridos. Se tomó muestras solo a las personas voluntarias que se acercaron a la campaña de tamizaje realizándose de este modo un tamizaje pasivo. Es importante mencionar que desde el momento de llegada a las diferentes comunidades se realizaron anuncios por radio y 
llamados por las calles con la ayuda de megáfonos, así como volantes promocionando la campaña de tamizaje que re realizaría. La recolección de datos fue realizada por personal del centro de Salud de Contamana de la provincia de Ucayali y a quienes previamente se capacitó (dirigido por equipo de ONG AHF Help Foundation ), el entrenamiento para una correcta relación con el poblador, toma de datos, toma muestras con las correctas medidas para evitar resultados falsos positivos, así como una correcta lectura del mismo y posteriormente una adecuada consejería post tamizaje duro 2 días y se llevaron a cabo en el centro de salud de Contamana. Asimismo, el personal contó con técnicos sanitarios de esta etnia que ayudaron en la traducción durante la toma de muestras y cuestionario, mas no traducción del documento en sí. Para el tamizaje de VIH se usaron los Rapid Test HIV 1/2 Ab Plus Combo Rapid Test (ARIA $\left.{ }^{\circledR}\right)$ y Rapid HIV I\&II Test (Lumiquick $\left.{ }^{\circledR}\right)$, las cuales tienen una sensibilidad y especificidad cercanas al 100\% (8)(11) (12). Para el presente proyecto de investigación se usaron 461 fichas de tamizaje completadas por el equipo de salud. Cada ficha contó con un identificador único.

\subsection{Análisis estadístico}

Los datos de las fichas de tamizaje fueron ingresados en una base de datos elaborada en Microsoft Access 2010. Posteriormente, se importaron al programa Microsoft Excel 2010 y se analizaron en el paquete estadístico STATA versión 14. Para asegurar la calidad de obtención de datos, se realizó una doble digitación. En cuanto al análisis descriptivo, las variables numéricas fueron expresadas en media y desviación estándar (DE); o mediana y rango intercuartil, dependiendo de la distribución de la variable. En cuanto a las categóricas, estas fueron expresadas en frecuencias y porcentajes. Para el análisis bivariado, se compararon variables numéricas usando $\mathrm{T}$ de Student o Prueba de Mann Whitney dependiendo de la frecuencia y distribución; para variables categóricas se utilizó Chi cuadrado o Prueba exacta de Fisher. Se consideró estadísticamente significativo un valor de $\mathrm{p}<0.05$.

Para el modelo multivariado inicial o modelo de regresión logística múltiple se incluyó a todas las variables significativas del estudio bivariado, posteriormente las variables no 
significativas fueron eliminándose sucesivamente del modelo empezando por aquellas con mayor valor de p (backward elimination) hasta lograr un modelo final en el que todas las variables presentaran un valor de $\mathrm{p}<0,05$. Se utilizó Odds Ratio (OR) como medida de asociación. Asimismo, se realizó modelos lineales generalizados de Poisson link log con varianza robusta, en el cual se utilizó Razón de Prevalencias (PR) como medida de asociación.

\subsection{Aspectos éticos}

El proceso de tamizaje y diagnóstico de la infección por el VIH en las comunidades indígenas es parte de las actividades ejecutadas por la estrategia de VIH en la Microred Ucayali. El protocolo de investigación fue realizado tomando como documento fuente las fichas de tamizaje empleadas por la Microred Ucayali, las que fueron aplicadas por el equipo de salud de Contamana. Al ser parte de una estrategia de tamizaje masiva, la toma de muestras, entrega de resultados, y la consejería pre y post test siguieron los procedimientos de la norma técnica de VIH. (13) El presente trabajo cuenta con la aprobación del Centro de Salud y por el comité de Ética de la Universidad Peruana de Ciencias Aplicadas. Los casos positivos fueron referidos a los establecimientos de salud correspondientes para iniciar terapia antirretroviral de acuerdo con las guías nacionales, trabajo realizado por el personal del programa de VIH de los respectivos centros. (13) 


\section{RESULTADOS}

4. Ampliar Resultados:

Se tamizaron a 461 pobladores de la etnia Shipibo-Konibo. Se observó que la población analizada es una población joven con una mediana de 27 (20-36,5), predominantemente de sexo femenino 295 (63.99\%), ejercen principalmente la agricultura o pescadería y el trabajo en casa 474 (102.82\%). La mayoría se encuentra en una relación (casado o conviviente) 325 (70.5\%) y con una frecuencia de consumo de alcohol de 129 (27.98\%) y tabaco 32 (6.94\%). La edad de inicio de relaciones sexuales es temprana, con una mediana de 15 años.

Se encontraron 53 casos de infección por VIH (11.49\%), no existieron pruebas discordantes, por ende, no hubo la necesidad de realizar carga viral durante el tamizaje. Se halló una mediana de edad de 24 años en comparación con una mediana de 27 en los pacientes VIH negativo, siendo la diferencia de edad estadísticamente significativa $(\mathrm{p}=0.038)$. Igualmente, la mediana de número de parejas sexuales fue mayor en el grupo de pacientes VIH positivo $2(1-2)$, sin alcanzar significancia estadística $(\mathrm{p}=0.931)$

Los pacientes VIH positivo presentaron una edad de inicio de relaciones sexuales menor (aunque sin significancia estadística) que aquellos pobladores VIH negativo (14 vs 15 años; $\mathrm{p}=0.159)$

Asimismo, se hallaron 45 gestantes, donde 12 (26,7\%) fueron casos VIH (+). El uso de sustancias toxicas como el alcohol fue significativamente mayor en los pobladores seropositivos al VIH que en los seronegativos (43.40\% vs $25.98 \%$; $=0.008$ ). La tabla 1 muestra las características de la población seropositiva y seronegativa.

El modelo de regresión logística múltiple inicial incluyó las variables significativas del estudio bivariado donde se incluyen: edad, orientación sexual, estado civil, conductas de riesgo (trabajador sexual, sexo con trabajador sexual, parejas eventuales), uso de alcohol, ITS como úlcera genital, manifestaciones clínicas como adenopatías, candidiasis, lesiones de Kaposi, diarrea y baja de peso. El modelo final incluyó la edad $(\mathrm{p}=0.002)$, presencia de diarrea $(p<0.001)$ y baja de peso $(p<0.001)$. (Tabla 2$)$

En el modelo de Regresión de Poisson con varianza robusta se incluyeron las mismas variables significativas que en el modelo de regresión logística múltiple. En el modelo final 
se observó que edad $(\mathrm{p}=0.001)$, presencia de diarrea $(\mathrm{p}<0.001)$ y baja de peso $(\mathrm{p}<0.001)$ permanecían siendo variable con significancia estadística. (Tabla 3) 


\section{DISCUSION}

La frecuencia de infección por VIH en la población Shipibo-Konibo de la Amazonía Peruana fue $11.49 \%$, siendo notoriamente mayor a la prevalencia general de VIH en el Perú, la cual es menor del $0.1 \%$ (14) (15). Nos encontramos frente a una epidemia generalizada, teniendo en cuenta el alto valor hallado de infección por VIH en gestantes (16). Aunque se suele considerar que la epidemia tiende a concentrarse en áreas urbanas (15), nuestros hallazgos son compatibles con una expansión de la enfermedad hacia áreas rurales, en un grupo poblacional altamente vulnerable. Esto es aún más preocupante, considerando que la población infectada con VIH es una población joven y con un inicio de vida sexual temprano, resultado concordante con los hallazgos en otras etnias amazónicas peruanas (1) (4). Lo cual, en presencia de barreras culturales al uso de métodos anticonceptivos de barrera, representa un riesgo de desarrollo hacia una epidemia en el corto y mediano plazo.

Dentro de las características que presenta esta población encontramos como posible factor asociado, la previamente mencionada (inicio sexual temprano). En promedio, el inicio sexual en los pacientes VIH (+) fue de 14 años, lo cual podría tener mayor importancia en cuanto a la cantidad de parejas sexuales que tienen a lo largo de la vida. Otro factor importante es la aceptación de relaciones sexuales de hombres con hombres sin identificarse como homosexuales. También se debe tener en cuenta que al ser comunidades ubicadas en la ribera del rio se presentan posibilidades de intercambio de parejas sexuales, sobre todo cuando presentan viajes que duran 2-3 días. Finalmente, un factor importante que aqueja a toda población de bajos recursos es la falta de información y conocimiento sobre la infección de VIH y la prevención del mismo (7). Por tal motivo, la comunidad no conoce los medios de transmisión, sintomatología ni factores asociados a la infección de VIH; y por otro lado la asocian a la infección de otras enfermedades endémicas como malaria y dengue (7).

Dos estudios peruanos han evaluado la frecuencia de infección por VIH en poblaciones indígenas amazónicas no pertenecientes al grupo Shipibo. En ellos se encontraron frecuencias de $7.5 \%$ y $0.7 \%$ (4) (17). Nuestros resultados se encuentran en una cifra superior a lo observado, posiblemente debido a que nos encontramos con una comunidad con mayor comercio y número de parejas sexuales. La variabilidad de las frecuencias observadas implica 
la necesidad de hacer estudios en diferentes comunidades para tener una idea cabal de la magnitud de la epidemia de infección por VIH en la Amazonía peruana.

Nuestro estudio es el primero en abordar la infección por VIH en la población Shipibo Konibo, por lo cual consideramos que nuestros datos son relevantes desde el punto de vista de salud pública y permiten una primera aproximación epidemiológica a la prevalencia de infección VIH en este grupo poblacional. Nuestros hallazgos sugieren una fuerte necesidad de implementar acciones inmediatas que no solo contribuyan a disminuir el riesgo de infección por VIH sino de otras infecciones de transmisión sexual. Para ello es importante adaptar las estrategias a la cultura y costumbres de esta comunidad.

En el análisis ajustado de nuestro estudio, evidenció como factor asociado a la infección de VIH la edad de los pobladores, siendo los más jóvenes quienes presentaban mayor riesgo de enfermedad. Asimismo, la diarrea y baja de peso fueron dos características fuertemente asociadas a la seropositividad, probablemente asociadas a un estadio clínico avanzado de la infección (SIDA). Es importante recalcar la alta frecuencia de gestantes infectadas $(27 \%$ del total de gestantes tamizadas), valores que nos hacen compararla con situaciones del continente Africano, donde se implementan medidas de prevención de infección vertical, debido a los altos porcentajes de muerte infantil a causa de la infección por VIH. Reportes del año 2010 en el África Subsahariana, indican cerca de 250,000 muertes de niños relacionadas con SIDA; por tal motivo decidieron adoptar la opción A de tratamiento de la OMS que indica profilaxis a las 14 semanas de gestación y la profilaxis diaria para el niño recién nacido hasta culminar con la etapa de la lactancia materna (18). Por tal motivo, consideramos importante que sería necesario realizar una evaluación profunda incluyendo un enfoque antropológico e intercultural probablemente en base a estudios con enfoque cualitativo con el objetivo de desarrollar estrategias de salud pública destinadas a abordar y evitar la propagación de la epidemia en esta población. Considerando que la mayoría de población son jóvenes sexualmente activos y probablemente con escaso acceso y escaso conocimiento al uso de métodos anticonceptivos como métodos de barrera, puede existir un alto potencial de diseminación de la enfermedad lo cual debe ser abordado por el Estado. 
Esto puede ser agravado por tratarse de grupos poblacionales donde se practica la poligamia (19) (20).

Las intervenciones sanitarias destinadas a abordar la problemática de la infección deben considerar las diferentes barreras sanitarias que presenta la comunidad Shipiba. Una de estas es la barrera cultural en donde el idioma y costumbres deben ser abordados de manera estratégica desarrollando planes en donde se use la lengua Shipiba y no interfiera en las costumbres de esta etnia. Otra barrera es la geográfica pues a muchas de estas comunidades solo se puede acceder por vía fluvial lo que dificulta la llegada del personal de salud. Otra, posiblemente sea la educación, pues es difícil llegar con mensajes de planificación familiar, enfocados en infecciones de transmisión sexual y métodos anticonceptivos de barrera.

Dentro de las limitaciones de nuestro estudio se encuentran el no haber contado con una muestra aleatoria debido a que la muestra fue limitada al número de personas tamizadas lo cual podría comprometer la representatividad de nuestros hallazgos y representar una sobreestimación. Asimismo, nuestros hallazgos podrían no ser generalizables a otras etnias amazónicas, en las que sería necesario realizar estudios para evaluar la real magnitud del problema. Por otro lado, existe una mínima posibilidad de resultados falsos positivos, esta cantidad podría aumentar si las pruebas no han sido conservadas adecuadamente, si no han sido conservadas en la temperatura adecuada $\left(4-30^{\circ} \mathrm{C}\right)$, si su lectura ha sido luego de 30 minutos de colocar la sangre total en la tarjeta del test. Es importante recalcar que este estudio utilizo OR como medida de asociación debido a que la frecuencia de VIH esperada era relativamente baja; sin embargo, la alta frecuencia encontrada (11.49\%) podría implicar que los OR hallados puedan ser mayores a las razones de prevalencia (RP), y así existir cierto grado de sobreestimación en los valores de OR. Al usar PR como medida de asociación se evidencio tal sobreestimación, aunque no se pueden comparar los valores en su totalidad; se observó que la variable edad presento OR de 0.927 y PR 0.950, lo mismo con Diarrea con OR 36.409 y PR 3.859 y con Baja de Peso con OR 12.771 y PR 4.629. Aunque los valore de OR y PR muestran diferencia cuantitativa, el sentido de las asociaciones es el mismo en ambos modelos. Nuestro estudio puede no tener la potencia necesaria para encontrar asociaciones, sin embargo, el objetivo primario fue evaluar la prevalencia y explorar los 
posibles factores asociados. Independientemente de estas consideraciones, la muy alta frecuencia de infección observada amerita la toma inmediata de acciones, el hallazgo más impórtate del presente estudio es la frecuencia de VIH, siendo necesario la toma de acciones no solo a nivel de los establecimientos de salud de las zonas evaluadas, sino posiblemente a nivel de todas las etnias amazónicas peruanas.

Consideramos estar frente a un riesgo latente de epidemia de VIH entre nativos ShipiboKonibo, teniendo como marcador de alerta importante la cantidad de pobladoras gestantes VIH (+). Es urgente contar con estudios que revelen la magnitud real de la infección a efectos de diseñar políticas de salud adaptadas al proceso cultural, tales como la fácil obtención de TARGA, impedir la transmisión vertical de la infección, y saber abordar adecuadamente a las madres VIH positivo que desean alimentar a sus hijos con lactancia materna no interfiriendo con su cultura pero si evitando la propagación de la enfermedad (7). También se debe tener en cuenta que las comunidades indígenas son poblaciones vulnerables, muchas de las cuales no cuentan con los servicios básicos o acceso rutinario a establecimientos de salud (21). Además, siendo una población joven se necesita fomentar el uso de preservativos y se debe investigar la estrategia adecuada para poder introducir su uso en la comunidad. 


\section{CONCLUSIONES}

En conclusión, nuestros datos evidencian una elevada frecuencia de infección por VIH en una población joven sexualmente activa desde muy temprana edad, lo que probablemente implica un alto riesgo de diseminación de la enfermedad en la población Shipibo-Konibo de la amazonia del Perú. Asimismo, se hallaron factores asociados significativos tales como edad, baja de peso y diarrea, siendo estos últimos partes de la clínica de una serie de patologías que se pueden presentar en áreas rurales, siendo importante el abordaje para la diferenciación de estas con un adecuado diagnóstico seguido de un oportuno tratamiento. Se necesitan más estudios para identificar la frecuencia de infección en distintas poblaciones amazónicas y desarrollar una estrategia de salud efectiva contra el riesgo de una posible epidemia de VIH adaptando las intervenciones a la cultura de ésta y otras etnias amazónicas.

\section{CONFLICTO DE INTERESES}

Los autores declaran que en la realización del artículo no existieron conflictos de intereses. 


\section{RECOMENDACIONES}

Se sugiere que se realice más estudios, los cuales abarquen mayor cantidad de población y mayores zonas geográficas. Tales estudios deben incluir investigaciones no solo de prevalencia sino también antropológicas pues al tratarse de comunidades indígenas se necesitan desarrollo de políticas de salud adecuadas a su medio y cultura. También se sugiere el posible desarrollo de cheklist para reconocer a pacientes con mayor probabilidad de infección de VIH o que incluso ya se encuentren en estadio SIDA. 


\section{Bibliografía}

1. Villalba JA, Bello G, Maes M, Sulbaran YF, Garzaro D, Loureiro CL, et al. HIV-1 epidemic in Warao Amerindians from Venezuela: spatial phylodynamics and epidemiological patterns. AIDS Lond Engl. 2013 Jul 17;27(11):1783-91.

2. South America :: Peru - The World Factbook - Central Intelligence Agency [Internet]. [cited 2018 Dec 10]. Available from: https://www.cia.gov/library/publications/the-world-factbook/geos/pe.html

3. Ormaeche M, Whittembury A, Pun M, Suárez-Ognio L. Hepatitis B virus, syphilis, and HIV seroprevalence in pregnant women and their male partners from six indigenous populations of the Peruvian Amazon Basin, 2007-2008. Int J Infect Dis IJID Off Publ Int Soc Infect Dis. 2012 Oct;16(10):e724-30.

4. Zavaleta C, Fernández C, Konda K, Valderrama Y, Vermund SH, Gotuzzo E. High prevalence of HIV and syphilis in a remote native community of the Peruvian Amazon. Am J Trop Med Hyg. 2007 Apr;76(4):7035.

5. Perú [Internet]. [cited 2018 Dec 24]. Available from: http://www.unaids.org/es/regionscountries/countries/peru

6. Shindo N, Alcantara LCJ, Van Dooren S, Salemi M, Costa MCR, Kashima S, et al. Human retroviruses (HIV and HTLV) in Brazilian Indians: seroepidemiological study and molecular epidemiology of HTLV type 2 isolates. AIDS Res Hum Retroviruses. 2002 Jan 1;18(1):71-7.

7. Orellana ER, Alva IE, Cárcamo CP, García PJ. Structural factors that increase HIV/STI vulnerability among indigenous people in the Peruvian amazon. Qual Health Res. 2013 Sep;23(9):1240-50.

8. IFU.HIV\&Self [Internet]. [cited 2019 Jan 15]. Available from: https://lumiquick.cld.bz/IFU-HIV-Self/5/

9. PRINCIPIO DE LA PRUEBA: USO PREVISTO: - PDF [Internet]. [cited 2019 Jan 15]. Available from: https://docplayer.es/5062719-Principio-de-la-prueba-uso-previsto.html

10. Delaney KP, Rurangirwa J, Facente S, Dowling T, Janson M, Knoble T, et al. Using a Multitest Algorithm to Improve the Positive Predictive Value of Rapid HIV Testing and Linkage to HIV Care in Nonclinical HIV Test Sites. J Acquir Immune Defic Syndr 1999. 2016 Jan 1;71(1):78-86.

11. Fransen K, de Baetselier I, Rammutla E, Ahmed K, Owino F, Agingu W, et al. Performance of serological and molecular tests within acute HIV infection. J Clin Virol Off Publ Pan Am Soc Clin Virol. 2017;93:81-4.

12. Masciotra S, Luo W, Westheimer E, Cohen SE, Gay CL, Hall L, et al. Performance evaluation of the FDAapproved Determine ${ }^{\mathrm{TM}}$ HIV-1/2 Ag/Ab Combo assay using plasma and whole blood specimens. J Clin Virol Off Publ Pan Am Soc Clin Virol. 2017;91:95-100.

13. Resolución Ministerial $\mathrm{N}^{\circ}$ 215-2018-MINSA [Internet]. [cited 2018 Dec 26]. Available from: https://www.gob.pe/institucion/minsa/normas-legales/187482-215-2018-minsa

14. Situación de la Epidemia de VIH en el Perú - PDF [Internet]. [cited 2018 Dec 10]. Available from: https://docplayer.es/19956319-Situacion-de-la-epidemia-de-vih-en-el-peru.html

15. Características sociodemográficas y del comportamiento sexual y reproductivo en adolescentes y jóvenes [Internet]. $\quad$ [cited 2018 Dec 10]. Available http://bvs.sld.cu/revistas/mgi/vol23_01_07/mgi07107.htm

16. [cited 2018 Dec 24]. Available from: http://www.unaids.org/sites/default/files/media_asset/JC2118_terminology-guidelines_es_0.pdf 
17. Bartlett EC, Zavaleta C, Fernández C, Razuri H, Vilcarromero S, Vermund SH, et al. Expansion of HIV and syphilis into the Peruvian Amazon: a survey of four communities of an indigenous Amazonian ethnic group. Int J Infect Dis. 2008 Nov 1;12(6):e89-94.

18. Hernández IB, Mapotere MJ. Efectividad de la eliminación de transmisión vertical de VIH en un centro de salud en Mozambique. Rev Cienc Médicas Pinar Río. 2017 Nov 1;21(6):936-42.

19. Hern WM. Polygyny and Fertility among the Shipibo of the Peruvian Amazon. Popul Stud. 1992 Mar 1;46(1):53-64.

20. Fuentes A. Porque las piedras no mueren: historia, sociedad y ritos de los chayahuita del Alto Amazonas. Lima: CAAAP; 1988. 235 p.

21. Cohen J. HIV/AIDS: Latin America \& Caribbean. Peru: universal access: more goal than reality. Science. $2006 \mathrm{Jul}$ 28;313(5786):489. 
Tabla 1: Características sociodemográficas de la población estudiada según status de seropositividad a VIH. Provincia de Ucayali, Perú. 2017 -2018

\begin{tabular}{|c|c|c|c|c|}
\hline Variable & Total $(n=461)$ & $V I H(+)(n=53)$ & $V I H(-)(n=408)$ & Valor de $p^{*}$ \\
\hline Edad & $27(20-36.5)$ & $24(20-29)$ & $27(20-36.5)$ & 0.038 \\
\hline Género Masculino & $166(36.01 \%)$ & $21(39.62 \%)$ & $145(35.54 \%)$ & $0.560\left(\mathrm{x}^{2}\right)$ \\
\hline Gestante & $45(9.78 \%)$ & $12(22.64 \%)$ & $33(8.11 \%)$ & $0.001\left(\mathrm{x}^{2}\right)$ \\
\hline $\begin{array}{l}\text { Edad de inicio de } \\
\text { relaciones sexuales }\end{array}$ & $15(14-16)$ & $14(14-15)$ & $15(13-16)$ & 0.159 \\
\hline \multicolumn{5}{|l|}{ Ocupación** } \\
\hline Agricultor/Pescador & $370(94.87 \%)$ & $34(97.14 \%)$ & $336(94.65 \%)$ & \multirow{4}{*}{$1.000(\mathrm{~F})$} \\
\hline Trabaja en casa & $3 / 0(94.8 / \%)$ & $34(91.14 \%)$ & $330(94.65 \%)$ & \\
\hline Comerciante & \multirow{2}{*}{$20(5.13 \%)$} & \multirow{2}{*}{$1(2.86 \%)$} & \multirow{2}{*}{$19(5.35 \%)$} & \\
\hline Campamento minero & & & & \\
\hline \multicolumn{5}{|l|}{ Estado civil } \\
\hline Conviviente & & & & \multirow{4}{*}{$0.001\left(\mathrm{x}^{2}\right)$} \\
\hline Casado & $325(10.5 \%)$ & $2 /(30.94 \%)$ & $298(13.04 \%)$ & \\
\hline Soltero & $136(295 \%)$ & $26(4906 \%)$ & $110(2696 \%)$ & \\
\hline Viudo & $136(29.5 \%)$ & $26(49.06 \%)$ & $110(26.96 \%)$ & \\
\hline \multicolumn{5}{|l|}{ Conductas de riesgo } \\
\hline Ninguna & $275(60.18 \%)$ & $16(30.19 \%)$ & $259(64.11)$ & \multirow{4}{*}{$<0.001\left(\mathrm{x}^{2}\right)$} \\
\hline Trabajador sexual & \multirow{3}{*}{$182(39.83 \%)$} & \multirow{3}{*}{$37(69.81)$} & \multirow{3}{*}{$145(35.89)$} & \\
\hline Sexo c/ trabajador sexual & & & & \\
\hline Parejas eventuales & & & & \\
\hline \multicolumn{5}{|l|}{ Consumo de tóxicos } \\
\hline Alcohol & $129(27.98 \%)$ & $23(43.40 \%)$ & $106(25.98 \%)$ & $0.008\left(\mathrm{x}^{2}\right)$ \\
\hline Tabaco & $32(6.94 \%)$ & $1(1.89 \%)$ & $31(7.6 \%)$ & $0.156(\mathrm{~F})$ \\
\hline Drogas & $1(0.22 \%)$ & $0(0 \%)$ & $1(0.25 \%)$ & $1.000(\mathrm{~F})$ \\
\hline \multicolumn{5}{|c|}{ ITS (Infecciones de transmisión sexual) } \\
\hline Ulcera genital & $25(5.42 \%)$ & $9(16.98 \%))$ & $16(3.92 \%)$ & $0.000\left(x^{2}\right)$ \\
\hline Descarga uretral & $17(3.69 \%)$ & $2(3.77 \%)$ & $15(3.68 \%)$ & $1.000(\mathrm{~F})$ \\
\hline Flujo vaginal & $113(24.51 \%)$ & $11(20.75 \%)$ & $102(25 \%)$ & $0.499\left(\mathrm{x}^{2}\right)$ \\
\hline Bubón Inguinal & $22(4.77 \%)$ & $3(5.56 \%)$ & $19(4.66 \%)$ & $0.730(\mathrm{~F})$ \\
\hline \multicolumn{5}{|l|}{ Manifestaciones Clínicas } \\
\hline Adenopatías & $7(1.52 \%)$ & $7(13.21 \%)$ & 0 & $<0.001(\mathrm{~F})$ \\
\hline Candidiasis oral & $10(2.17 \%)$ & $8(15.9 \%)$ & $2(0.49 \%)$ & $<0.001(\mathrm{~F})$ \\
\hline Hepatoesplenomegalia & $1(0.22 \%)$ & 0 & $1(0.25 \%)$ & $1.000(\mathrm{~F})$ \\
\hline Lesiones de Kaposi & $1(0.22 \%)$ & $1(1.89 \%)$ & 0 & $0.115(\mathrm{~F})$ \\
\hline Diarrea & $25(5.42 \%)$ & $23(43.40 \%)$ & $2(0.49 \%)$ & $<0.001(\mathrm{~F})$ \\
\hline Baja de Peso & $34(7.38 \%)$ & $25(47.17 \%)$ & $9(2.21 \%)$ & $<0.001\left(\mathrm{x}^{2}\right)$ \\
\hline \multicolumn{5}{|l|}{ Orientación sexual } \\
\hline Heterosexual & $397(86.12 \%)$ & $36(67.92 \%)$ & $361(88.48 \%)$ & \multirow{3}{*}{$<0.001\left(\mathrm{x}^{2}\right)$} \\
\hline Homosexual & & & & \\
\hline Bisexual & $64(13.89 \%$ & $17(32.08 \%)$ & $47(11.52 \%)$ & \\
\hline Número de parejas & $1(1-2)$ & $2(1-2)$ & $1(1-2)$ & 0.931 \\
\hline
\end{tabular}


$(*)$ Wilcoxon signed Rank test para variables numéricas y Prueba de Chi2 o prueba exacta de Fisher para categóricas.

$\left.{ }^{(* *}\right)$ Los porcentajes pueden sumar más de $100 \%$ en caso de personas con más de una ocupación.

$\left(x^{2}\right)$ Se utilizo Chi2 para la asociación de variables categóricas

(F) Se utilizo Prueba exacta de Fisher para la asociación de variables categóricas

Tabla 2 : Modelo multivariado de variables significativas, Provincia de Ucayali, Perú. 2017.(*)

\begin{tabular}{|c|c|c|c|c|c|c|}
\hline & \multicolumn{3}{|c|}{ Análisis multivariado inicial } & \multicolumn{3}{|c|}{ Modelo multivariado final } \\
\hline & OR & (IC95\%) & $\begin{array}{l}\text { Valor } \\
\text { de } p\end{array}$ & OR & (IC95\%) & $\begin{array}{l}\text { Valor } \\
\text { de p }\end{array}$ \\
\hline Edad & 0.892 & $(0.832-0.957)$ & 0.002 & 0.927 & $(0.884-0.973)$ & 0.002 \\
\hline $\begin{array}{l}\text { Estado Civil (sin } \\
\text { pareja vs unido) }\end{array}$ & 1.393 & $(0.607-3.119)$ & 0.434 & \multicolumn{3}{|c|}{ No incluida } \\
\hline $\begin{array}{c}\text { Presencia de } \\
\text { Conductas de } \\
\text { riesgo }\end{array}$ & 2.008 & $(0.853-4.730)$ & 0.110 & \multicolumn{3}{|c|}{ No incluida } \\
\hline $\begin{array}{c}\text { Consumo de } \\
\text { Alcohol }\end{array}$ & 2.089 & $(0.789-5.530)$ & 0.138 & \multicolumn{3}{|c|}{ No incluida } \\
\hline Ulcera Genital & 0.069 & $(0.003-1.211)$ & 0.067 & \multicolumn{3}{|c|}{ No incluida } \\
\hline Candidiasis & 0.470 & $(0.010-21.342)$ & 0.698 & \multicolumn{3}{|c|}{ No incluida } \\
\hline Diarrea & 60.849 & $(6.494-570.119)$ & $<0.001$ & 36.409 & $(6.789-195.238)$ & $<0.001$ \\
\hline Baja de peso & 19.485 & $(4.671-81.274)$ & $<0.001$ & 12.771 & $(3.899-41.831)$ & $<0.001$ \\
\hline $\begin{array}{c}\text { Orientación sexual } \\
\text { (homosexual/bisexual } \\
\text { Vs heterosexual) }\end{array}$ & 1.069 & $(0.351-3.258)$ & 0.905 & \multicolumn{3}{|c|}{ No incluida } \\
\hline
\end{tabular}

(*) Se realizo regresión logística multivariada 
Tabla 3 : Modelo multivariado de variables significativas, Provincia de Ucayali, Perú. 2017.(**)

\begin{tabular}{|c|c|c|c|c|c|c|}
\hline & \multicolumn{3}{|c|}{ Análisis multivariado inicial } & \multicolumn{3}{|c|}{ Modelo multivariado final } \\
\hline & $P R$ & (IC95\%) & $\begin{array}{l}\text { Valor } \\
\text { de p }\end{array}$ & $P R$ & (IC95\%) & $\begin{array}{l}\text { Valor } \\
\text { de p }\end{array}$ \\
\hline Edad & 0.935 & $0.904-0.966$ & $<0.001$ & 0.950 & $0.929-0.972$ & $<0.001$ \\
\hline $\begin{array}{l}\text { Estado Civil (sin } \\
\text { pareja vs unido) }\end{array}$ & 1.133 & $0.675-1.901$ & 0.635 & \multicolumn{3}{|c|}{ No incluida } \\
\hline $\begin{array}{l}\text { Presencia de } \\
\text { Conductas de } \\
\text { riesgo }\end{array}$ & 1.605 & $0.874-2.947$ & 0.127 & \multicolumn{3}{|c|}{ No incluida } \\
\hline $\begin{array}{l}\text { Consumo de } \\
\text { Alcohol }\end{array}$ & 1.334 & $0.729-2.443$ & 0.349 & \multicolumn{3}{|c|}{ No incluida } \\
\hline Ulcera Genital & 0.693 & $0.413-1.161$ & 0.164 & \multicolumn{3}{|c|}{ No incluida } \\
\hline Candidiasis & 0.803 & $0.378-1.702$ & 0.568 & \multicolumn{3}{|c|}{ No incluida } \\
\hline Diarrea & 3.392 & $1.418-8.114$ & 0.006 & 3.859 & $1.726-8.626$ & 0.001 \\
\hline Baja de peso & 3.947 & $1.543-10.091$ & 0.004 & 4.629 & $2.007-10.674$ & $<0.001$ \\
\hline $\begin{array}{c}\text { Orientación sexual } \\
\text { (homosexual/bisexual } \\
\text { Vs heterosexual) }\end{array}$ & 1.153 & $0.619-2.149$ & 0.651 & \multicolumn{3}{|c|}{ No incluida } \\
\hline
\end{tabular}

(**) Se empleó regresion de Poisson 
Figura 1: Flujograma para diagnóstico de infección por VIH, Ucayali, Loreto-Perú 2017

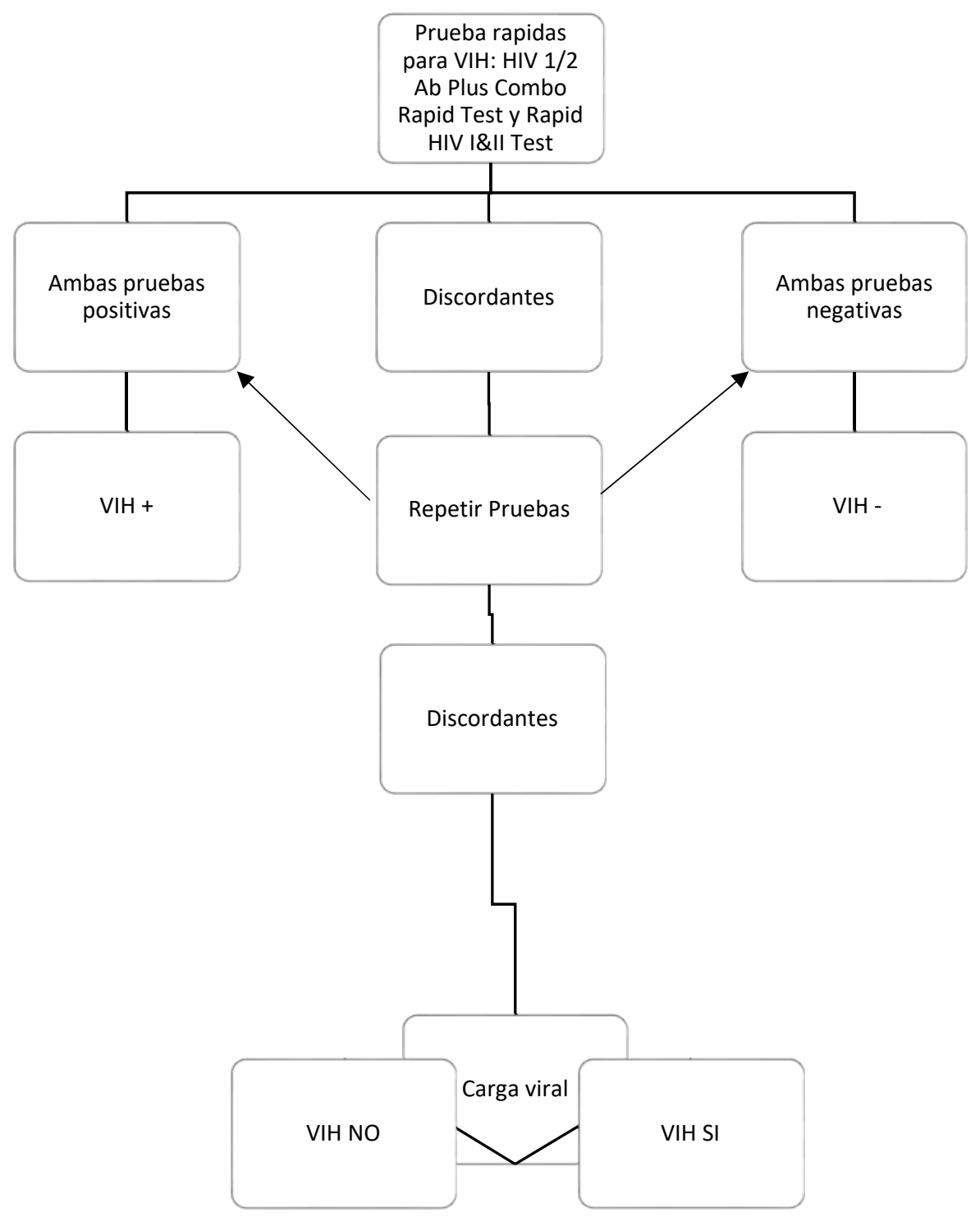




\title{
APROBACIONES:
}

\author{
- Centro de Salud de Contamana
}

Contamana, 12 de Abril del 2017

Doctor

Aldo Javier Lucchetti Rodriguez

Profesor de la Facultad de Medicina Humana

de la Universidad Peruana de Ciencias Aplicadas.

Presente-

Asunto: Autorización para el uso de fichas de Tamizaje del Cetro de Salud Contamana.

Estimado Doctor,

Es grato dirigirme a usted con la finalidad de saludarlas y a la vez informarles que luego de hacer lectura de su protocolo de Investigaçión titulado "Frecuencia de infección por VIH en comunidades Shipibo - Konibo de la región Loreto", desarrollado infección por VIH en comunidades Shipibo - Konibo de la reg por sus alumnas, las señoritas MARYCIELO volicitud antes enviada al Centro de Salud, HAYASHIDA, otorgamos el permiso a la solicitud antes enviada al Centro de salud realizadas en Contamana, las mismas que podrán ser utilizadas por ustedes en su proyecto de investigación desarrollado en el curso Proyecto de Tesis 1 y 2.

Sin otro particular y agradeciendo la atención prestada, quedamos de ustedes.

Atentamente,

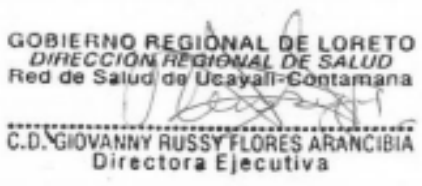


Ficha de evaluación

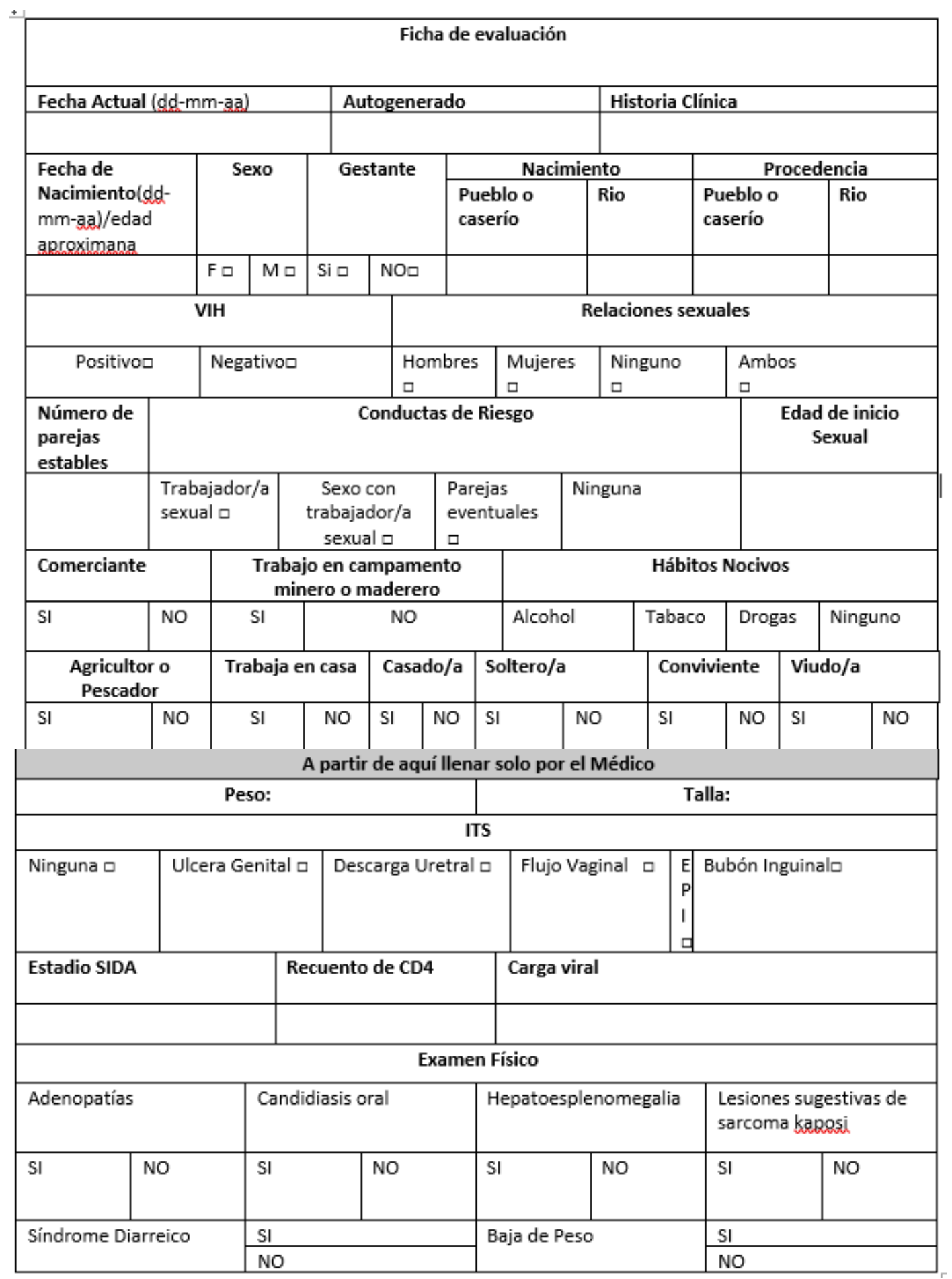


Consentimiento Informado, Programa de Tamizaje VIH, MINSA

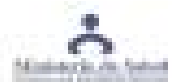

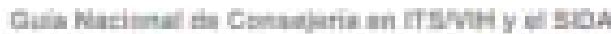

Pit mari

Miringrie de Ealut

\section{ANEXO 11}

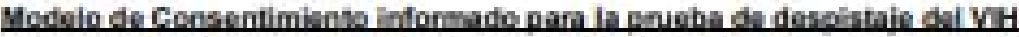

Ho,

ostifios

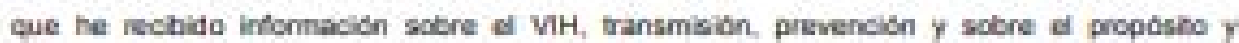

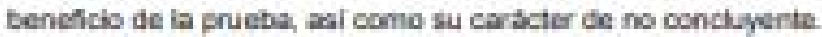

Ou he recibdo bonsebria pre test: para prepararme y combotame con relacion a mis

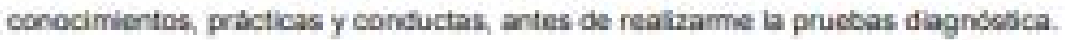

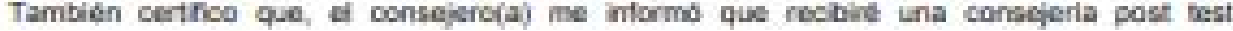

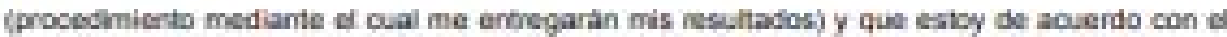
procese

Endento que la toma de mustra sa vountera, y que puobo rether $m$ consentmiens en

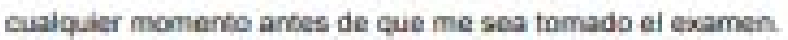

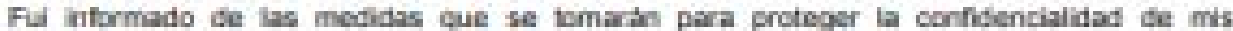
resultros:

Nombra y frma de quben da di conchimkento

Nombre Fuma

Fima dol probsionsl que roulas ta oonicheria Nonbre Fima

Fecha

Lugar 